\title{
Etude Des Plantes Médicinales Utilisées Par Les Femmes Autochtones Pygmées (Batwa) Enceintes Dans L'hinterland Du Parc National De Kahuzi-Biega (Rift albertin, RD. Congo)
}

\section{Mukumba Isumbisho Pacifique,}

Doctorant à l'Ecole Doctorale de l'Université du Burundi, Chercheur au Centre de Recherche pour la Promotion Rurale (CERPRU) de l'Institut Supérieur de Développement Rural de Bukavu (ISDR-Bukavu), Bukavu RD. Congo

\section{Mangambu Mokoso Jean De Dieu,}

Professeur à l'Université Officielle de Bukavu, Département de Biologie, Laboratoire de Systématique, Biodiversité et Management des Ecosystèmes, Bukavu, RD. Congo

\section{Iragi Kaboyi Gentil,}

Chercheur au Centre de Recherche en Sciences Naturelles CRSN/Lwiro, Département de Biologie, section Botanique, laboratoire d'Ethnobotanique Bukavu, RD. Congo

\section{Manirakiza René,}

Professeur à l'Université du Burundi,Bujumbura, Burundi

\section{Doi:10.19044/esj.2020.v16n27p107 URL:http://dx.doi.org/10.19044/esj.2020.v16n27p107}

\section{Résumé}

Introduction : La femme devient vulnérable à certaines pathologies pendant la grossesse en milieux ruraux et le recours à la pharmacopée traditionnelle en utilisant les plantes médicinales est la solution souvent adoptée. Cet article se focalise sur l'utilisation des plantes médicinales chez les femmes autochtones Batwa enceintes vivant aux alentours du Parc National de Kahuzi Biega (PNKB). Les connaissances et les pratiques de ces femmes nous ont permis d'inventorier et de déterminer les différents usages de ces plantes.

Méthodologie : Les données utilisées dans cette étude ont été collectée au moyen d'une enquête ethnobotanique menée à l'aide d'un questionnaire en suivant la méthode de l'interview semi-structuré. Les données collectées qui portent sur les caractéristiques ethnobotaniques, ont été traitées et analysées 
avec des logiciels ethnobotaniques et statistiques appropriés afin de sélectionner quelques plantes pour de futurs tests de laboratoire.

Résultat : La plupart des tradipraticiens interviewés étaient des femmes $(83,88 \%)$ et une grande proportion d'entre eux (35\%) était âgée de plus de 65 ans. Les plantes utilisées ont été classées en fonction des différents stades de l'évolution de la grossesse. Ainsi, les Dichrocephala integrifolia, Kalanchoe pinnata, Pennicetum purpureum Plantago palmata,Commelina difusa , Physalis angulata, Aloe barbadensis, Rumex bequaertii, Cannabis sativa, Carapa grandiflora Myrianthus arborea et Acmella caulirhiza ont un taux de fidélité de $100 \%$. Les organes les plus utilisés sont des tiges feuillées $(37,14 \%)$ et les pressages $(66,6 \%)$ sont les plus citées en tant que techniques de préparation.

Conclusion : Ces résultats illustrent la richesse de ces peuples et ces ressources végétales méritent un intérêt scientifique en vue d'une bonne valorisation.

Mots clés : Savoirs traditionnels, Populations autochtones, Plantes médicinales, Ressources naturelles. 


\section{Study of Medicinal Plants Used by Indigenous Pygmy (Batwa) Women Pregnant in the Hinterland of Kahuzi-Biega National Park (Albertine Rift, DR. Congo)}

\section{Mukumba Isumbisho Pacifique,}

Doctorant à l'Ecole Doctorale de l'Université du Burundi, Chercheur au

Centre de Recherche pour la Promotion Rurale (CERPRU) de l'Institut Supérieur de Développement Rural de Bukavu (ISDR-Bukavu), Bukavu RD.

Congo

\section{Mangambu Mokoso Jean De Dieu,}

Professeur à l'Université Officielle de Bukavu, Département de Biologie, Laboratoire de Systématique, Biodiversité et Management des Ecosystèmes,

Bukavu, RD. Congo

\section{Iragi Kaboyi Gentil,}

Chercheur au Centre de Recherche en Sciences Naturelles CRSN/Lwiro, Département de Biologie, section Botanique, laboratoire d'Ethnobotanique

Bukavu, RD. Congo

Manirakiza René,

Professeur à l'Université du Burundi,Bujumbura, Burundi

Abstract

Introduction: Women become vulnerable to certain pathologies during pregnancy in rural areas and the use of traditional pharmacopoeia using medicinal plants is the solution often adopted. This article focuses on the use of medicinal plants among pregnant indigenous Batwa women living around the Kahuzi Biega National Park (KBNP). The knowledge and practices of these women have enabled us to inventory and determine the different uses of these plants.

Methodology: The data used in this study was collected by means of an ethnobotanical survey conducted using a questionnaire following the semistructured interview method. The data collected, which included ethnobotanical characteristics, were processed and analyzed with appropriate ethnobotanical and statistical software in order to select a few plants for future laboratory testing.

Result: Most of the traditional practitioners interviewed were women $(83.88 \%)$ and a large proportion of them (35\%) were over 65 years of age. The plants used were classified according to the different stages of pregnancy 
development. Thus, Dichrocephala integrifolia, Kalanchoe pinnata, Pennicetum purpureum, Plantago palmata, Commelina difusa, Physalis angulata , Aloe barbadensis, Rumex bequaertii, Cannabis sativa, Carapa grandiflora Myrianthus arborea and Acmella caulirhiza have a $100 \%$ fidelity. The most commonly used organs are leafy stems (3714\%) and pressing (66, $6 \%$ ) is the most cited preparation technique.

Conclusion: These results illustrate the richness of these peoples and these plant resources deserve scientific interest in order to be properly valorized.

Keywords: Traditional knowledge, Indigenous peoples, Medicinal plants, Natural resources.

\section{Introduction}

$\mathrm{Au}$ cœur de la forêt, les Pygmées établissent leurs campements constitués de huttes, construites souvent par des femmes, ou de bivouacs en fonction de leur projet de séjour et surtout en fonction de la générosité de la nature (Mubalama et al, 2018). Ils s'installent durablement généralement près d'un ruisseau et sous des arbres de plusieurs mètres de hauteur.

Un campement Pygmée abrite une famille avec des logis différemment conçus pour les ménages et pour les célibataires. Les jeunes filles et les jeunes garçons vivent séparément (Mangambu et Kambale, 2019). Dans la forêt, les populations pygmées s'adonnent à des activités qui leur procurent les produits nécessaires à leur alimentation. Ils tirent toutes leurs ressources de cette forêt en menant des activités de chasse, de cueillette et parfois de pêche.

Aujourd'hui, le soucis de préservation de l'environnement suivi par des activités de protection de la nature avec la création de plusieurs aires ou réserves protégées ont poussé les peuples autochtones Pygmées à changer de mode de vie en vivant dans les villages avec les autres peuples et en recourant à l'élevage (Mangambu et Kambale, 2019). Malgré cette nouvelle vie, ils font toujours référence et recourent à leur vie traditionnelle. Ils pratiquent la médecine traditionnelle et vivent souvent de la chasse, de la cueillette et du ramassage. Ils expliquent que, durant des siècles et même des millénaires, leurs ancêtres ont utilisé les plantes pour soulager leurs douleurs, guérir leurs maux et panser leurs blessures. Ainsi, les peuples autochtones vivant dans l'hinterland du Parc National de Kahuzi-Biega recourent toujours à ces pratiques (Mangambu et al., 2012 et 2015a). De génération en génération, ils se sont transmis leur savoir et leurs expériences en s'efforçant quand ils le pouvaient de les consigner par écrit. Bien que la pharmacologie moderne a fait des progrès, l'usage thérapeutique des plantes médicinales est toujours présent dans cette communauté.

Tabuti et al., (2003) montrent que cette pratique est plus utilisée chez les indigènes des pays en voie de développement, en l'absence d'un système 
médical moderne adéquat. Par ailleurs, d'après nos observations, les femmes des pygmées autour du PNKB fréquentent rarement les centres de santé et les hôpitaux pour leurs consultations prénatales ou pour leurs accouchements.

Leurs maris affirment détenir des remèdes traditionnels très efficaces pour pallier aux problèmes d'accouchement et de traitement pendant la grossesse. Ils nous ont informé et l'avons même constaté que les autres ethnies de cet hinterland du PNKB usent des savoirs des Pygmées dans l'utilisation des plantes médicinales afin de pallier aux difficultés d'accès aux soins de santé maternelle et infantile.

Selon l'OMS, la grossesse constitue l'état physiologique de la femme depuis la fécondation jusqu'à l'accouchement (OMS, 2017). Tout au long de cette période qui dure en moyenne neuf mois, la femme subit de nombreuses transformations physiologiques qui lui permettent de porter, de protéger et d'assurer le développement et la croissance du fœtus qu'elle porte. Aussi du fait de ce changement d'état durant cette période, elle devient vulnérable à certaines pathologies. Pour cette raison, il est important que toutes les grossesses soient suivies par des agents de santé qualifiés (OMS, 2017). Malheureusement, les femmes de la zone d'étude ne bénéficient pas de ce suivi suite à l'absence d'infrastructure de santé, de personnel qualifié et de moyens pour accéder aux soins de santé adéquats.

Toutefois, d'après Bafor (2017), les plantes médicinales peuvent être utilisées pour leurs effets bénéfiques sur de nombreux processus de reproduction chez la femme, depuis la grossesse jusqu'à l'induction d'accouchement, l'élimination de la rétention placentaire et la gestion de l'hémorragie post-partum. Depuis quelques décennies, des organismes internationaux comme l'Organisation des Nations Unies pour l'Alimentation et l'Agriculture (FAO), la Banque Mondiale et l'Organisation des Nations Unies pour l'Education, la Science et Culture (UNESCO), tendent cependant à reconnaître les vertus thérapeutiques de ces plantes pour des soins des femmes enceintes (Pourchez, 2011).

Selon Gururani (2002), les femmes pauvres des zones rurales possèdent un savoir-faire lié à leur mode de subsistance, à leur corps et à leur environnement naturel et c'est évident qu'elles ont acquis une connaissance élaborée en plantes médicinales. Cet avis a été confirmé par Ahmed et al. (2018).

D'après ces auteurs, les traitements par les plantes sont connus et pratiqués en Afrique depuis longtemps, car ces savoirs sont transmis de génération en génération. Approximativement, $80 \%$ de la population africaine utilisent les plantes pour résoudre leurs problèmes de santé. Et les femmes enceintes utilisent fréquemment de médicaments à base des plantes médicinales, mais les effets néfastes de ces espèces végétales les plus utilisées sur le fœtus pendant la grossesse, ne sont pas connus. 
A l'aide des enquêtes ethnobotaniques, cette étude va documenter sur les plantes que les femmes enceintes Pygmées usent pour leurs soins pendant la grossesse et l'accouchement. En deuxième lieu, cette étude veut établir un lien entre les espèces des plantes médicinales des maladies soignées chez les femmes autochtones Batwa enceintes.

Son intérêt sera de montrer l'utilité des plantes médicinales chez les femmes Batwa de la zone d'étude dans le suivi de la grossesse. Il y a peu de travaux sur les plantes médicinales et cette étude pourra contribuer dans la promotion des savoirs notamment en mettant en lumière la part de ces plantes dans la prise en charge de la grossesse et de l'accouchement chez les Batwa de la zone étudiée.

Parmi le peu de travaux dans le domaine réalisé dans cette zone, on peut citer le lexique et le recueil de quelques pratiques en ethno-pharmacopée agrovétérinaire du Kivu réalisé par l'ONG plateforme Diobas en 2005, les catalogues des plantes médicinales rudérales ou cultivées de Defour (1995), Schneider (1996) et Balagizi et al. (2007). D'autres auteurs qui travaillé dans le domaine sont Mangambu et al. (2012 et 2015a\&b) qui ont étudié l'ethnomédecine et la conservation des ptéridophytes autour de la forêt des montagnes du PNKB ainsi que l'ethnobotanique et ethnolinguistique des ressources forestières ligneuses utilisées par la population du couloir écologique de ce Parc. Shalukoma et al. (2015) ont aussi effectué une étude sur les plantes médicinales de la région montagneuse de ce Parc en se basant sur l'utilisation, l'accessibilité et le consensus des tradipraticiens. Le présent article approfondit les connaissances concernant les usages thérapeutiques de ces plantes.

\section{Méthode et matériels}

\section{Description de la zone d'étude}

Le Parc National de Kahuzi Biega (PNKB, Figure 1), vaste d'environ $600 \mathrm{Km} 2$, se trouve à l'Est de République Démocratique du Congo (RDC) dans la partie Sud de la chaîne Kivu-Ruwenzori et se localise entre les deux centres d'endémisme guinéo-congolais et afro-montagnard (Mangambu, 2013).

Il tire son nom de deux montagnes (Kahuzi : 3326 mètres et Biega : 2790 mètres) qui dominent sa partie de haute altitude (Mangambu, 2016). Ce parc enregistre une présence remarquable de Gorille de Grauer (Gorilla beringei graueri, Chibembe et al., 2015). C'est un site du patrimoine mondial de l'UNESCO depuis 1980, mais, malheureusement, depuis 1996, il est inscrit sur la liste du patrimoine mondial en péril.

Selon leur physionomie et leur composition floristique, en fonction de l'altitude, les forêts du PNKB se différencient en étages planétaire (678 -1250 $\mathrm{m})$, sub montagnard $(1250-1700 \mathrm{~m})$, montagnard $(1700-2600 \mathrm{~m})$ et afro 
subalpin (2600 - 3326 mètres) (Mangambu, 2013). Cet article se focalise sur l'hinterland de la partie dite " moyenne et haute altitudes" du Parc (Figure1). Elle se présente sous forme d'une bande allongée le long de la forêt équatoriale (Mangambu, 2013 et Imani et al, 2016). Dans cette zone submontagnarde, la densité de troncs y est plus forte, mais le volume ligneux est inférieur (200$300 \mathrm{~m}^{3} /$ ha ; Mangambu, 2013).

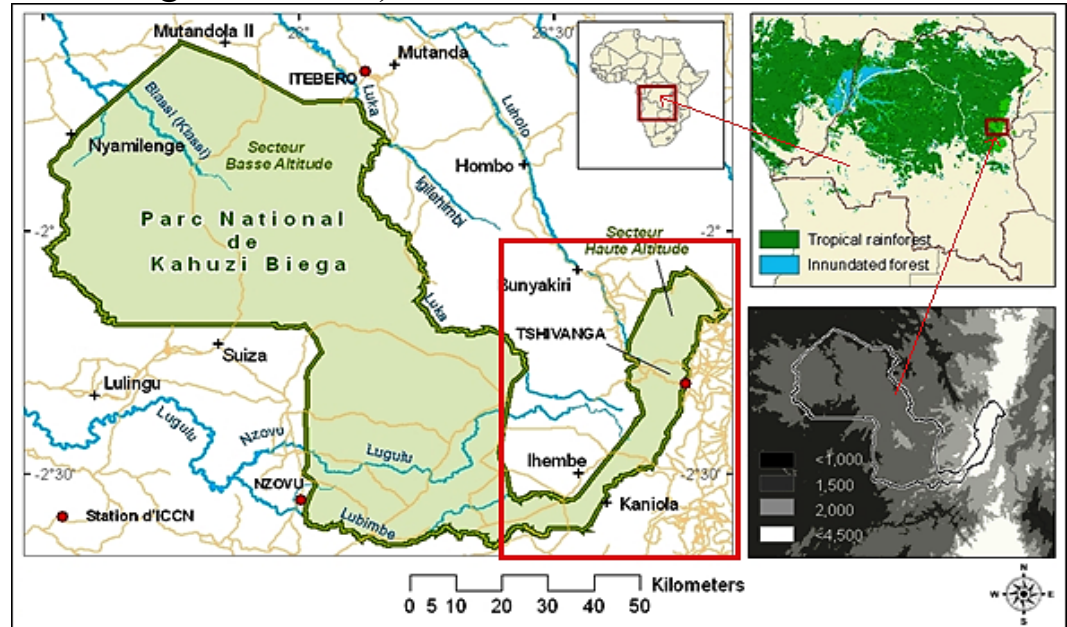

Figure 1 : Localisation du site de recherche dans l'hinterland de la haute altitude du PNKB (Amisi et al., 2008, modifié).

Dans la zone purement des montagnes qui apparait au-delà de 1700 mètres, la végétation est hétérogène à sous-bois très touffu, contrairement à la forêt équatoriale typique avec la présence des groupements à Bambou. Les épiphytes (Ptéridophytes et des Orchidées) sont particulièrement abondants. Au dela de $2600 \mathrm{~m}$ jusqu'à $3326 \mathrm{~m}$, la végétation est caractérisée par la présence des Ericaceae et des bruyères sur les sommets des monts Kahuzi et Biega.

Dans son ensemble, cette partie étudiée bénéficie du climat de montagne (du type Cf de Köppen), avec des pluies abondantes, oscillant entre 1750-2000 mm par an. La durée de la saison sèche ne dépasse pas deux mois. La température varie selon l'altitude, et le sol est superficiel et acide (Mangambu, 2013).

Collecte des données

La méthode utilisée est celle de l'ethnobotanique quantitative selon la technique d'enquête ethnobotanique. Cette enquête consiste à constituer un questionnaire (Bernstein1997 ; Kamini 2007 et Maregesi et al., 2007), permettant d'appréhender la connaissance et l'usage des plantes médicinales par les femmes enceintes Pygmées pour se soigner (De Caluwé et al., 2009 ; Malay, 2011). 
La collecte des données s'est effectuée par la méthode des entretiens semi-structurés (Kakudidi, 2004, Mangambu et al., 2015a). Les données ont été principalement collectées dans quatre villages situés dans les territoires de Kabare et de Kalehe. Dans le territoire de Kabare, les enquêtes ont été réalisées dans les villages Batwa de Chombo et Tshibati. Nos enquêtés parlent les langues « Shi » et « Kiswahili ». Alors qu'à Kalehe, la collecte s'est déroulée dans les villages de Hungu et Kainga, dans le groupement de Bitale. Les enquêtés de ce goupement parlent les langues «Tembo » et « Kiswahili ». Le choix de ces villages a été consécutif à la présence des peuples Batwa et surtout celle des femmes Batwa en âge de procréer.

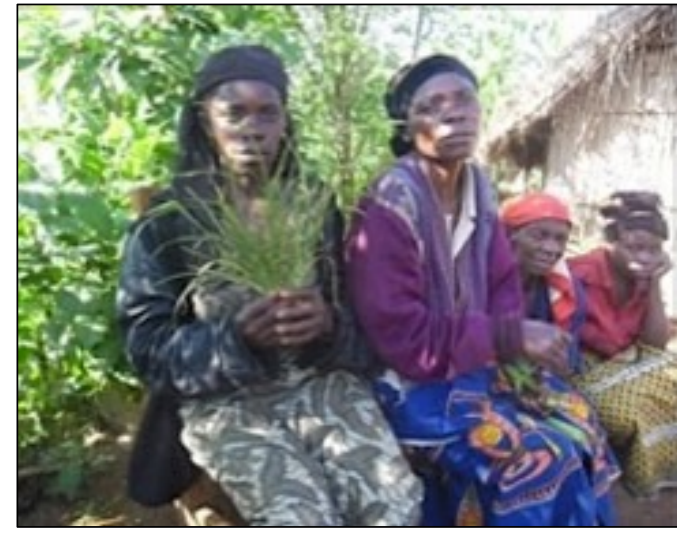

Figure 2 : Enquête à Bitale

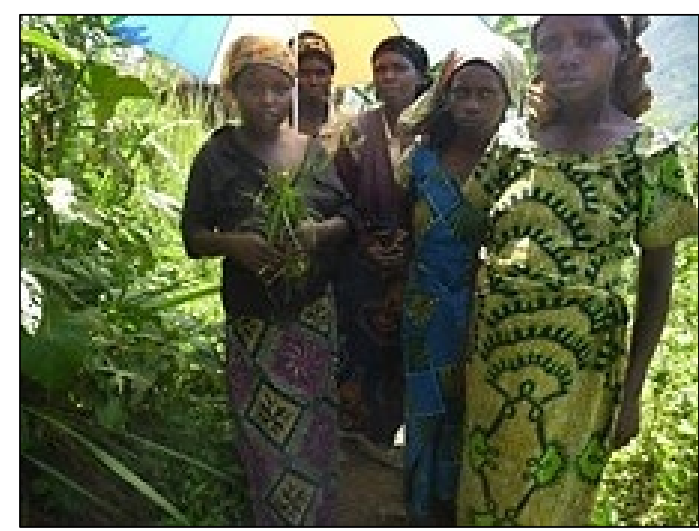

Figure 3 : Récolte des échantillons sur terrain

\section{Identification}

Les identifications des espèces récoltées sur terrain ont été vérifiées à la fois par comparaison avec les spécimens de référence conservés dans l'herbier du Centre de Recherches en Sciences Naturelles de Lwiro (LWI), de l'INERA Mulungu (MLGU) et dans celui du Jardin Botanique National de Belgique (BR). Les noms des plantes à fleurs ont été vérifiés à l'aide de travaux de Lebrun et Stork (1991- 2012) et de Bloesch et al. (2009) et par les systèmes basés sur l'approche phylogénétique tenant compte des données de la botanique systématique moléculaire (APG IV, 2016).

\section{Analyse des données}

Pour atteindre les résultats, nous avons effectué les analyses ci-après : - Taux de réponse (TR) : Nous avons utilisé le test de Maregesi et al. (2007) pour calculer le taux de réponse tel qu'exprimé par cette équation :

$$
\operatorname{Tr}=100 \frac{\mathrm{S}}{\mathrm{N}}
$$

Où, Tr: taux de réponse calculé ; $\mathrm{S}:$ nombre de personne ayant donné une réponse positive (Oui) pour l'utilisation de l'organe concerné ; $\mathrm{N}$ : nombre 
total de personnes interviewées. Il indique les organes les plus utilisés pour chaque espèce dans le milieu et varie de 0 à 100 .

- Niveau de connaissance des plantes par les femmes: Le niveau de connaissance des plantes médicinales a été évalué par la Fréquence de Citation (FC). La FC est le nombre de fois que le répondant a mentionné une plante donnée. C'est un bon indice pour évaluer la crédibilité des informations reçues et le niveau de connaissance des plantes au sein d'une population. Ce paramètre, pour chaque plante, a été déterminé par la formule de (Tardío \& Pardo-de-Santayana, 2008) :

$$
F C=\frac{\text { Nombre de citation }}{\text { Nombre total de citation }} \times 100
$$

- Niveau d'utilisation des plantes médicinales par les femmes : Le niveau d'utilisation des plantes médicinales a été évalué par la Fréquence de consommation(Fi). La Fi est le nombre moyen de fois qu'un individu utilise une espèce par mois. Elle permet de connaître la consommation mensuelle ou quotidienne et a été estimée selon Yao et al.(2015) :

$$
\mathrm{F}_{\mathrm{i}}=\frac{\Sigma \frac{f_{i}}{n}}{30} \times 100
$$

Avec fi : Fréquence de consommation individuelle, n, nombre de fois qu'un individu i consomme une espèce donnée par mois.

- Accord sur l'utilisation des plantes medicinales : L'accord sur l'utilisation des plantes medicinales a été évaluées par le Facteur Consensuel de l'Informateur (ICF). L'ICF montre le degré de consensus sur une information concernant l'utilisation d'une espèce pour une maladie donnée. Il est utile pour apprécier les accords des informateurs sur les thérapies rapportées pour chaque catégorie d'utilisation (Heinrich et al., 1998). Il est déterminé par la formule :

$$
\mathrm{ICF}=\frac{\mathrm{Nur}-\mathrm{Nt}}{\mathrm{Nur}-1}
$$

avec Nur : le nombre de fois qu'une catégorie de maladie a été mentionnée et $\mathrm{Nt}$ : le nombre de plante(s) mentionnée(s) pour son traitement. Ainsi, quand l'ICF est supérieur à 0,5 , le degré de consensus est élevé pour l'information. Au contraire, lorsqu'il est inférieur à 0,5 , le degré de consensus est faible.

- Niveau de Fidélité(NF) : Le NF est un paramètre qui repose sur la concordance des réponses des informateurs pour une indication thérapeutique donnée (Friedman et al., 1986).

$$
N F=\frac{\mathrm{Np}}{N}
$$

Avec $\mathrm{Np}$ : le nombre d'informateurs qui mentionnent une espèce pour un certain usage $\mathrm{p} ; \mathrm{N}$ : le nombre d'informateurs qui mentionnent l'espèce pour n'importe quel usage. 
- Importance culturelle des plantes médicinales dans la communauté des Batwa : l'mportance culturelle des plantes medicinales dans la communauté a été évaluée par l'Indice Culturel d'Importance (IC). L'IC, qui traduit l'importance chiffrée d'une plante dans un milieu, est la somme des proportions d'informateurs qui mentionnent chaque espèce. Il est utile pour apprécier la signification culturelle de chaque taxon (Tardío \& Pardo-deSantayana, 2008) et est déterminé par la formule :

$$
\text { IC }=\sum_{U=U 1}^{U N C} \sum_{I=1}^{I N} \frac{U R u i}{N}
$$

Avec URui : Nombre d'informateurs ayant mentionnée l'usage ; N : Nombre total d'informateurs ; NC : Nombre de catégories d'usages

\section{Résultats}

\section{Données socio - démographiques}

L'étude socio - démographique indique que, dans les ménages, 83,88\% des enquêtés sont des femmes et $16,12 \%$ sont des hommes (Tableau 1). Parmi les professions enregistrées, les plus représentées sont les ménagères $(51,57 \%)$, suivies des agricultrices-ménagères $(40,72 \%)$ et des agriculteurs $(5,65 \%)$. La classe d'âges la plus représentée est celle des personnes de plus de 65 ans (35\%) suivie des autres adultes dont l'intervalle d'âges est compris entre 30-45 ans (34\%). Le traitement des données nous a permis de constater que les femmes aux tranches d'âge [21-30] avec 55\% viennent en première position dans l'utilisation des plantes médicinales suivies par celles de 31 ans et plus avec $28 \%$. La classe la moins utilisatrice est celle des jeunes femmes âgées de 18 à 20 ans avec $12 \%$ d'utilisatrices.

La connaissance des usages des plantes médicinales et leurs propriétés sont généralement acquises suite à une longue expérience accumulée dont l'âge constitue la principale source d'information à l'échelle locale chez ce peuple. La majorité des personnes interviewées ont un âge supérieur à 45 ans $(80,77 \%)$. Le poids de l'âge (Khi2 $=20,171 ; \mathrm{P} \leq 0,0001)$ et le sexe $(\mathrm{Khi} 2=$ $4,5594 ; \mathrm{p} \leq 0,05)$ influencent significativement la pratique de la médecine traditionnelle. 
Tableau 1 : Données socio- démographiques des ménages des Batwa dans l'hinterland du Parc National de Kahuzi-Biega

\begin{tabular}{|c|c|c|c|}
\hline \multirow[b]{2}{*}{ Paramètres } & \multicolumn{3}{|c|}{ Territoire } \\
\hline & Kabare & Kalehe & Total \\
\hline Effectif & 203 & 284 & 487 \\
\hline Sexe $(\%)$ & & & Moyenne \\
\hline Masculin & 10,52 & 21,71 & 16,12 \\
\hline \multirow[t]{2}{*}{ Féminin } & 89,48 & 78,29 & 83,88 \\
\hline & 100 & 100 & 100 \\
\hline \multicolumn{4}{|l|}{ Profession (\%) } \\
\hline Ménagères & 50,74 & 52,41 & 51,58 \\
\hline Commerçants & 0,75 & 1,01 & 0,88 \\
\hline Artisans & 1,93 & 0,41 & 1,17 \\
\hline Agriculteurs Ménager(ères) & 38,32 & 43,13 & 40,72 \\
\hline \multirow[t]{2}{*}{ Agriculteurs (trices) } & 8,26 & 3,04 & 5,65 \\
\hline & 100 & 100 & 100 \\
\hline \multicolumn{4}{|c|}{ Classe d'âges (\%) } \\
\hline$<30$ & 11 & 8 & 9,5 \\
\hline$[30-45]$ & 25 & 18 & 21,5 \\
\hline$[46-65]$ & 30 & 38 & 34 \\
\hline \multirow[t]{2}{*}{$>65$} & 34 & 36 & 35 \\
\hline & 100 & 100 & 100 \\
\hline \multicolumn{4}{|c|}{ Tranche d'âges d'usage des plantes médicinales (\%) } \\
\hline$[18-20]$ & 16 & 18 & 17 \\
\hline$[21-30]$ & 56 & 54 & 55 \\
\hline \multirow[t]{2}{*}{$>31$} & 28 & 28 & 28 \\
\hline & 100 & 100 & 100 \\
\hline
\end{tabular}

\section{Niveau de connaissance des plantes par les femmes batwa}

Dans les villages parcourus dans les deux territoires (Kabare et Kalehe), les taux de connaissance des plantes médicinales des femmes enceintes Batwa sont élevés. Ils dépassent plus de $75 \%$ selon la population autochtone interrogée. Le taux le plus élevé $(91 \%)$ est enregistré à Kalehe dans le village Hungu. Dans l'ensemble des villages visités dans l'hinterland $\mathrm{du}$ PNKB, le taux de personnes connaissant cette usage pendant la grossesse représente $85,5 \%$ de la population interrogée (Tableau 2 ).

Tableau 2 : Distribution des taux de connaissance d'usage des plantes médicinales pendant la période prénatale.

\begin{tabular}{|c|c|c|c|}
\hline \multirow[t]{2}{*}{$\begin{array}{l}\text { Localités } \\
\text { visitées }\end{array}$} & \multirow{2}{*}{$\begin{array}{l}\text { Nombre de } \\
\text { personnes } \\
\text { interrogées }\end{array}$} & $\begin{array}{c}\text { Personnes connaissant } \\
\text { la pratique }\end{array}$ & $\begin{array}{l}\text { Personnes ne connaissant } \\
\text { pas cette pratique }\end{array}$ \\
\hline & & Nombre & Nombre \\
\hline
\end{tabular}




\begin{tabular}{|c|c|c|c|c|c|}
\hline Kabare & & & & & \\
\hline Chombo & 100 & 81 & $81 \%$ & 19 & $19 \%$ \\
\hline Tshibati & 100 & 79 & $79 \%$ & 21 & $21 \%$ \\
\hline Sous total & 200 & 160 & 80 & 40 & $20 \%$ \\
\hline \multicolumn{6}{|l|}{ Kalehe } \\
\hline Hungu & 100 & 94 & $94 \%$ & 06 & $6 \%$ \\
\hline Kainga & 100 & 88 & $88 \%$ & 12 & $12 \%$ \\
\hline Sous total & 200 & 182 & $91 \%$ & 18 & $9 \%$ \\
\hline Total Général & 400 & 342 & 85,5 & 58 & 14,5 \\
\hline
\end{tabular}

\section{Richesse floristique}

Trente-cinq (35) espèces de plantes médicinales utilisées par des femmes autochtones Batwa réparties entre 34 genres et 22 familles ont été inventoriées. Les familles les plus représentées sont les Asteraceae (8 espèces, soit 34,37\%) et les Lamiaceae (16,62 espèces) suivies des Fabaceae, Poaceae, Rubiaceae et Solanaceae avec 2 espèces, soit 9,09\% (Tableau 3). Parmi ces espèces inventoriées, la forme morphologique herbeuse est la plus utilisée et représente 26 espèces différentes (soit 74,3\%) suivie des arbustes (5 espèces, soit $14,3 \%$ ) tel que repris au tableau 3 .

L'exploitation de la médecine traditionnelle des femmes autochtones Batwa enceintes ne cause pas de préjudices aux plantes. Les feuilles et les tiges récoltées sont les organes des plantes médicinales qui sont les plus utilisés pour traiter les maladies. Leur fréquence estimée est de $35,7 \%$ pour les tiges et les feuilles $31 \%$.

Tableau 3 : Espèces médicinales recensées chez les femmes Batwa (Noms scientifiques et vernaculaires, les familles d'appartenance et les types morphologiques)

\begin{tabular}{lllll}
\hline $\mathbf{N}^{\mathbf{0}}$ & N. Scie. & N.Ver. & Fml. & TM \\
\hline 1 & Acmella caulirhiza & Tshenda & Asteraceae & Her. \\
2 & Aframomum laurenti & Mitero & Zingiberaceae & Her. \\
3 & Ageratum conyzoides & Chitende & Asteraceae & Her. \\
4 & Aloe barbadensis & Chizimyamuliro & Agavaceae & Her. \\
5 & Bidens pilosa & Kashisha & Asteraceae & Her. \\
6 & Bothriocline longipes & Lwibaye & Asteraceae & Her. \\
7 & Cannabis sativa & Irumba & Cannabaceae & Her. \\
8 & Carapa grandiflora & Eweji & Meliaceae & Arb. \\
9 & Commelina difusa & Mutesha & Commelinaceae & Her. \\
10 & Conyza sumatrensis & Nyambuba & Asteraceae & Her. \\
11 & Crassocephalum bumbens & Kifubula & Asteraceae & Her. \\
12 & Crassocephalum vitellinum & Chichununu & Asteraceae & Her. \\
13 & Crotalaria sp & Karanga ka basimu & Fabaceae & Her. \\
14 & Dichrocephala integrifolia & Chitundambuga & Asteraceae & Her. \\
15 & Dioscorea minutiflora & Birongo & Dioscoreaceae & Her. \\
16 & Eleusina indica & Chisesa & Poaceae & Her. \\
17 & Embelia pelucida & Mulanzo & Myrsinaceae & L. \\
18 & Gladiolu spsittacinus & Buku & Iridaceae & Her. \\
19 & Kalanchoe pinnata & Ineke & Crassulaceae & Her. \\
20 & Leucas deflexa & Kanyamafundo & Lamiaceae & Her.
\end{tabular}




\begin{tabular}{ll}
21 & Musa sempervirens \\
22 & Myrianthus arborea \\
23 & Ocimum gratissimum \\
24 & Pauridiantha bequaertii \\
25 & Pennicetum purpureum \\
26 & Physalis angulata \\
27 & Pseudospondia smicrocarpa \\
28 & Piper guineense \\
29 & Newtonia buchananii \\
30 & Plantagopalmata \\
31 & Raphia farinifera \\
32 & Rumex bequaertii \\
33 & Solanum nigrum \\
34 & Spermacoce princeae \\
35 & Tetradenia riparia \\
\hline
\end{tabular}

Mboko
Byambamba
Kausu
Lubiku
Masheke
Itunda
Ngobia
Lunjululu
Lukundu
Chikungwa
Bubondo
Kabera
Milunda
Chomomo
Mutuzo

Musaceae

Her.

Urticaceae Arb.

Lamiaceae Suf.

Rubiaceae Arb.

Poaceae Her.

Solanaceae Her.

Anacardiaceae Arb.

Piperaceae Her.

Fabaceae A

Plantaginaceae Her.

Arecaceae Arb.

Polygonaceae Her.

Solanaceae Her.

Rubiaceae Her.

Légende : 1). Noms scientifiques (N.Scie); Noms Vernaculaires (N.Scie.); Familles (Fml.) et Types morphologiques (T.M) ; 2). Arbre : A ; Arbuste : Arb. ; Herbe : Her ; Sous arbuste : S-arb. ; Suffritex : Suf.

\section{- Pathologies traitées et recettes utilisées pendant la période prénatale}

D'une manière générale, nous avons regroupé les pathologies et les modes de traitement par des analyses statistiques tels que illustrés dans les tableaux quatre jusqu'au tableau numéro huit. Ces tableaux ci-dessus illustrent le niveau de connaissance des plantes par les femmes, le niveau d'utilisation des plantes médicinale par les femmes, l'accord sur l'utilisation des plantes médicinale, le taux de réponse ainsi que l'importance culturelle des plantes médicinales dans la communauté

\section{- Métrorragie sans dilatation du col (Menace d'avortement)}

Les plantes utilisées dans le traitement des menaces d'avortement sont cosignées dans le tableau 4 ci-dessous.

Tableau 4 : Traitement des menaces d'avortement

\begin{tabular}{|c|c|c|c|c|c|c|c|c|}
\hline Espèce végétale & Organe utilisé & Préparation & $\mathrm{NC}$ & $\mathrm{FiC}$ & FRC & $\mathrm{IC}$ & $\mathrm{Tr}$ & $\mathrm{NF}$ \\
\hline *1Dichrocephala integrifolia & Feuilles et tige & Pressage & 76 & 8,90 & 0,16 & 0,48 & 19 & 100 \\
\hline Crotalaria sp & Tiges feuillées & Pressage & 61 & 7,02 & 0,12 & 0,60 & $\begin{array}{c}15,2 \\
5\end{array}$ & 86 \\
\hline Eleusina indica & Tiges feuillées & Macération & 51 & 4,37 & 0,01 & 0,18 & $\begin{array}{c}12,7 \\
5\end{array}$ & 75,6 \\
\hline Kalanchoe pinnata & Feuilles & Pressage & 52 & 4,95 & 0,02 & 0,05 & 13 & 100 \\
\hline Musa sempervirens & Feuille terminale & Décoction & 12 & 0,40 & 0,02 & 0,48 & 3 & 42,1 \\
\hline Pauridiantha bequaertii & Feuilles & Macération & 20 & 1,02 & 0,01 & 0,04 & 5 & 62,7 \\
\hline Pennicetum purpureum & Jeunes feuilles & Macération & 38 & 1,37 & 0,00 & 0,05 & 9,5 & 100 \\
\hline Plantago palmata & Plante entière & Pressage & 41 & 2,57 & 0,01 & 0,04 & $\begin{array}{c}10,2 \\
5\end{array}$ & 100 \\
\hline Raphia farinifera & Jeunes feuilles & Décoction & 27 & 1,18 & 0,02 & 0,15 & 6,75 & 65 \\
\hline Tetradenia riparia & Feuilles & Macération & 19 & 0,98 & 0,02 & 0,15 & 3,25 & 55 \\
\hline
\end{tabular}


$\mathrm{NC}$ : nombre de citation ; FC : fréquence de citation ; FRC : Fréquence Relative de citation ; Fi : Fréquence de Consommation; TR : Taux de réponse, NF : Niveau de Fidélité d'usage des plantes médicinales

*1 Recette bispécifique faisant intervenir, en association l'espèce Bidens pilosa

\section{- Anorgasmie, douleurs avant accouchement et accouchement}

Dans le tableau 5 ci-dessous sont consignés les modes d'usages des maladies telles que l'anorgasmie et les douleurs avant accouchement.

Tableau 5 : Traitement de frigidité et douleurs avant accouchement

\begin{tabular}{|c|c|c|c|c|c|c|c|c|}
\hline Espèce végétale & $\begin{array}{l}\text { Organe } \\
\text { utilisé }\end{array}$ & $\begin{array}{c}\text { Préparati } \\
\text { on }\end{array}$ & $\begin{array}{l}\mathrm{N} \\
\mathrm{C}\end{array}$ & $\mathrm{FiC}$ & $\begin{array}{c}\text { FR } \\
\text { C }\end{array}$ & $\mathrm{IC}$ & $\mathrm{Tr}$ & $\mathrm{NF}$ \\
\hline \multicolumn{9}{|c|}{ Anorgasmie } \\
\hline *2Embelia pelucida & $\begin{array}{c}\text { Tiges } \\
\text { feuillées }\end{array}$ & Pressage & 64 & 7,12 & $\begin{array}{c}0,6 \\
0\end{array}$ & $\begin{array}{l}4, \\
55\end{array}$ & 16 & 95 \\
\hline \multicolumn{9}{|c|}{ Dilatation } \\
\hline $\begin{array}{l}\text { Crassocephalum } \\
\text { crepidioides }\end{array}$ & $\begin{array}{c}\text { Tiges } \\
\text { feuillées }\end{array}$ & Pressage & 61 & 7,02 & $\begin{array}{c}0,1 \\
3 \\
\end{array}$ & $\begin{array}{l}0 \\
62 \\
\end{array}$ & $\begin{array}{c}15,2 \\
5 \\
\end{array}$ & 86 \\
\hline \multicolumn{9}{|c|}{ Accouchement } \\
\hline Commelina difusa & Tige & Pressage & $\begin{array}{c}13 \\
2 \\
\end{array}$ & $\begin{array}{c}13,9 \\
0\end{array}$ & 5,3 & $\begin{array}{l}0 \\
48\end{array}$ & 33 & 100 \\
\hline
\end{tabular}

*2 Recette bispécifique faisant intervenir, en association avec le vin de banane

\section{- Malaria et Anémie}

Le mode de traitement du paludisme et la diminution pathologique du nombre de globules rouges ou du taux d'hémoglobine dans le sang chez les peuples Batwa se présente dans le tableau 6 ci-dessous.

Tableau 6 : Traitement du paludisme et de la souffrance d'anémie

\begin{tabular}{ccccccccc}
\hline Espèce végétale & Organe utilisé & Préparation & NC & FiC & FRC & IC & Tr & NF \\
\hline & & Paludisme & & & & & & \\
\hline Biden spilosa & Tiges feuillées & Pressage & 75 & 8,02 & 0,08 & 3,31 & 18,75 & 89 \\
\hline Aloe barbadensis & Feuilles & Pressage & 100 & 10,02 & 0,05 & 4,31 & 14,75 & 100 \\
\hline Bothrioclin elongipes & Tiges feuillées & Infusion & 43 & 2,58 & 0,18 & 5,88 & 10,75 & 94,12 \\
\hline *3 Physalis angulata & Feuilles & Pressage & 135 & 13,71 & 0,04 & 0,01 & 8,75 & 100 \\
\hline & & Anémie & & & & & & \\
\hline Crassocephalum vitellinum & Tiges feuillées & Décoction & 115 & 9,40 & 0,1 & 0,51 & 28,75 & 3,8 \\
\hline Pseudospondias microcarpa & Fruits & Pressage & 114 & 9,40 & 0,09 & 0,48 & 28,5 & 3,5 \\
\hline
\end{tabular}

*3 Recette trispécifique faisant intervenir, en association Physalis plus Bothriocline

- Constipation, douleurs après accouchement et maux de ventre créant des troubles de la grossesse

Les autres types des maladies telles que constipation, les maux de ventre se présentent dans le tableau 7 ci-dessous, tel que recensé sur le terrain.

Tableau 7 : Traitement de constipation, les maux de ventre créant troubles de la grossesse et des douleurs après accouchement

\begin{tabular}{lllllllll} 
Espèce végétale & Organe utilisé & Préparation & NC & FiC & FRC & IC & Tr & NF \\
& & Constipation & & & & & & \\
\hline
\end{tabular}




\begin{tabular}{|c|c|c|c|c|c|c|c|c|}
\hline Aframomum laurenti & Fruits & Pressage & 12 & 0,40 & 0,00 & 0 & 3 & 58 \\
\hline \multicolumn{9}{|c|}{ Maux de ventre } \\
\hline $\begin{array}{l}\text { Ageratum } \\
\text { conyzoides }\end{array}$ & Tiges feuillées & Pressage (Lavement) & 12 & 0,40 & 0,00 & 0 & 3 & 75 \\
\hline Cannabis sativa & Tiges feuillées & infusion & 15 & 0,42 & 0,00 & 0,51 & 3,8 & 68 \\
\hline Rumex bequaertii & Plante entière & Décoction & 13 & 0,40 & 0,00 & 0,48 & 3,3 & 100 \\
\hline Solanum nigrum & Feuilles & Pressage & 12 & 0,40 & 0,00 & 0 & 3 & 82 \\
\hline \multicolumn{9}{|c|}{ Expulsion d'un fotus, naturelle ou provoquée, avant son terme de viabilité } \\
\hline Acmellacaulirhiza & $\begin{array}{l}\text { Fleurs et } \\
\text { feuilles }\end{array}$ & Pressage & 132 & 13,9 & 5,3 & 1,48 & 33 & 100 \\
\hline
\end{tabular}

*4 Recette bispécifique faisant intervenir le mélangée avec la cendre de Celosia

\section{- Autres maladies}

Les autres différents types des recettes pour traiter les pathologies se trouvent dans le tableau 8.

Tableau 8 : Traitement d'autres types de maladies inventoriées

\begin{tabular}{|c|c|c|c|c|c|c|c|c|}
\hline Espèce végétale & Organe utilisé & Préparation & $\overline{\mathrm{NC}}$ & $\mathrm{FiC}$ & FRC & IC & $\operatorname{Tr}$ & $\mathrm{NF}$ \\
\hline \multicolumn{9}{|c|}{ Maux d'estomac } \\
\hline Cannabis sativa & Tiges feuillées & infusion & 13 & 0,40 & 0,00 & 0,48 & 3,3 & 100 \\
\hline *5Carapa grandiflora & fruits & Cendre & 13 & 0,40 & 0,00 & 0,48 & 3,3 & 100 \\
\hline Myrianthus arborea & Fruits & Pressage & 35 & 3,01 & 0,04 & 0,01 & 8,75 & 100 \\
\hline \multicolumn{9}{|c|}{ Angines } \\
\hline *6Conyza sumatrensis & Tiges feuillées & Pressage & 41 & 2,40 & 0,00 & 0,51 & 3,75 & 62,3 \\
\hline Spermacoce princeae & Feuilles & Pressage & 14 & 0,40 & 0,00 & 0,48 & 3,5 & 71,3 \\
\hline \multicolumn{9}{|c|}{ Anorexie (Manque d'appétit) } \\
\hline Dioscorea minutiflora & Fruits & Pressage & 15 & 0,40 & 0,01 & 0,51 & 3,75 & 62,3 \\
\hline \multicolumn{9}{|c|}{ Diarrhée } \\
\hline *7Leucas deflexa & Plantes entières & Pressage & 37 & 3,06 & 0,04 & 0,02 & 9,25 & 100 \\
\hline \multicolumn{9}{|c|}{ Malaise } \\
\hline Ocimum gratissimum & Tiges feuillées & Pressage & 12 & 0,40 & 0,00 & 0 & 3 & 85 \\
\hline \multicolumn{9}{|c|}{ Dysenterie } \\
\hline Piper guineense & $\begin{array}{c}\text { Tigesfeuillesplus } \\
\text { les fruits }\end{array}$ & Pressage & 55 & 4,40 & 0,00 & 0 & 13,25 & 75 \\
\hline \multicolumn{9}{|c|}{ Vertiges (Indisposition) } \\
\hline Newtonia buchananii & Ecorces & Pressage & 51 & 3,19 & 0 & 0,13 & 12,25 & 83,33 \\
\hline
\end{tabular}

*5 Recette mélangée avec de l'eau, *6 Recette mélangée avec le sel, *7Recette bispécifique faisant intervenir une association avec le Cannabis sativa

Selon les informations recueillies, diverses utilisations des plantes ont été mentionnées dans les différents tableaux présentés. Des plantes sont utilisées pour le bon développement du fœtus et luttent contre le traitement de menace d'avortement. Elles représentent 28,5\% dans l'ensemble des inventaires. $12,28 \%$ des plantes répertoriées sont utilisées dans le traitement et la prévention de certaines pathologies comme le paludisme et la constipation (douleurs abdominales). D’autres plantes sont associées dans la préparation en remèdes dans les cas des grossesses, par exemples Dichrocephala integrifolia est mélangée avec l'espèce Bidens pilosa pour 
traiter les menaces d'avortement (tableau 4), Leucas deflexa est associée avec le Cannabis sativa pour traiter la diarrhée (tableau 8)

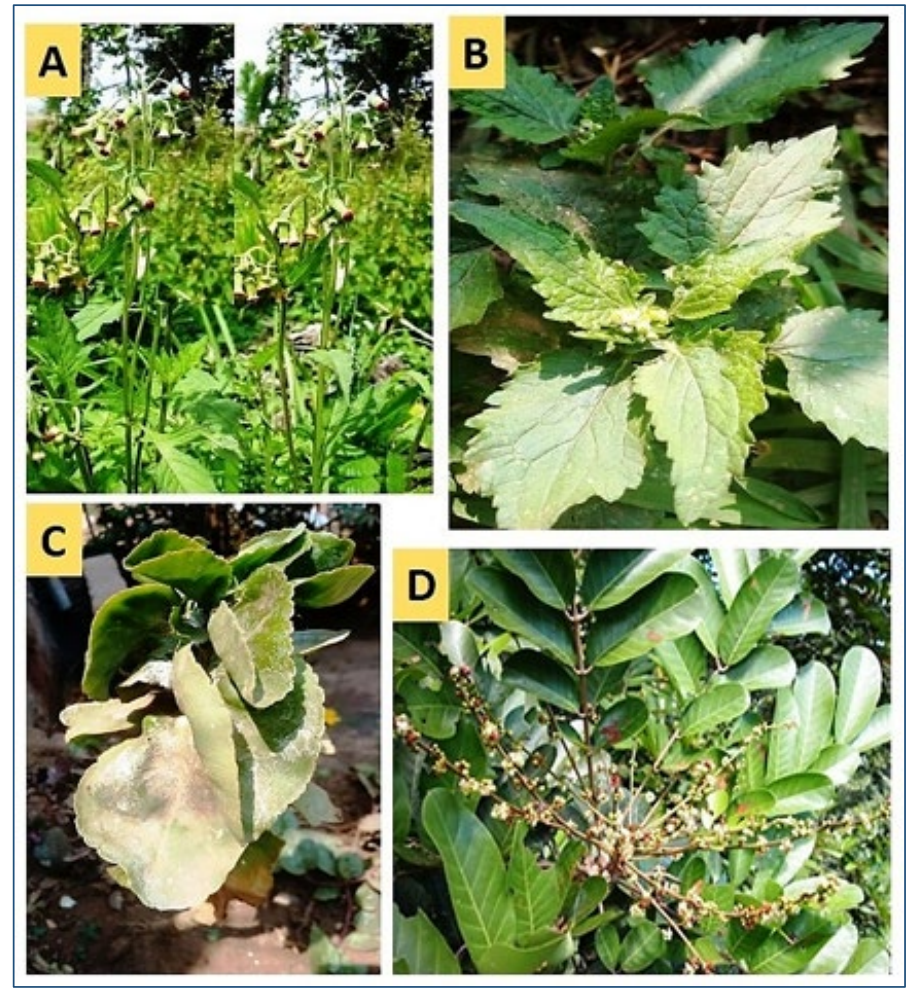

Figure 4 : Quelques plantes médicinales utilisées par les femmes autochtones 'Batwa' (A : Crassocephalum crepidioides B : Dichrocephala integrifolia, C : Kalanchoe pinnata, D : Carapa grandiflora)

Ces tableaux (4-8) montrent aussi le taux de réponse lié à l'utilisation des 35 récentes d'espèces inventoriées auprès de ces 487 enquêtés. Il ressort de ces tableaux 4 jusqu'à 8 que ces espèces sont utilisés par les enquêtés et on note une certaine variabilité des réponses d'une espèce à une autre. D'une manière générale, le taux de réponse, le nombre de citation, la fréquence de citation ; la fréquence relative de citation, la fréquence de consommation, le niveau de fidélité sont plus élevés chez certaines espèces tel que Dichrocephala integrifolia, Kalanchoe pinnata, Pennicetum purpureum Plantago palmata pour le traitement de menace d'avortement avec un taux de fidélité de $100 \%$.

Pour d'autres pathologies, certaines autres espèces tel que Commelina difusa, Physalis angulata, Aloe barbadensis, Rumex bequaertii, Cannabis sativa, Carapa grandiflora, Myrianthus arborea et Acmella caulirhiza ont aussi un taux de fidélité de $100 \%$ ainsi que d'autres paramètres statistiques calculés. Ces résultats montrent que les recettes ci-haut citées satisfont les femmes autochtones qui les utilisent. Néanmoins, certaines autres espèces 
malgré les valeurs parfois insignifiantes, ont aussi une forte utilisation dans l'un ou l'autre village.

\section{Préparations et formes d'utilisation des plantes}

- Parties utilisées

La figure 5 ci-dessus illustre que les tiges feuillées (13 espèces, soit 37, 14 $\%$ ) et feuilles ( 5 espèces, soit $20 \%$ ) sont les organes des plantes médicinales les plus utilisés pour traiter les maladies et les maux liés à la grossesse. Les analyses statistiques nous montrent que $\mathrm{R}^{2}$ est inférieure à 0,5 et le $\mathrm{P}$-value $>0,05$ qui montre les résultats fiables. Ceci s'expliqué les autres organes des plantes sont moins utilisés par rapport à l'usage des tiges feuillées qui est le mieux utilisées par ce peuple.

Toutefois, en connaissant l'importance de la plante, les organes sensibles de la plante comme les racines et les écorces ainsi que les plantes entières ne sont pas beaucoup utilisés par les Batwa pour pérenniser la vie des plantes. Ce mode d'exploitation par les autochtones Batwa de la zone d'étude, ne cause pas de préjudice aux plantes.

\section{- Modes de préparation des recettes}

Les résultats des modes de préparation des recettes tel que présenté dans la figure 6 montrent que le pressage des plantes vient en première position (24 recettes, soit $66,6 \%$ ). Les résultats des autres modes de préparation des recettes sont fiables (figure 6).

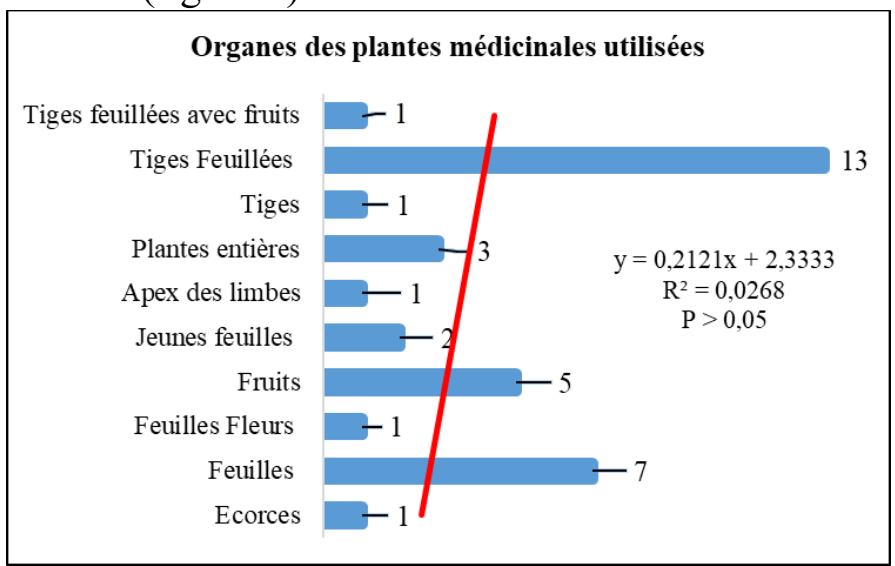

Figure 5 : Utilisations des organes des plantes récoltées 


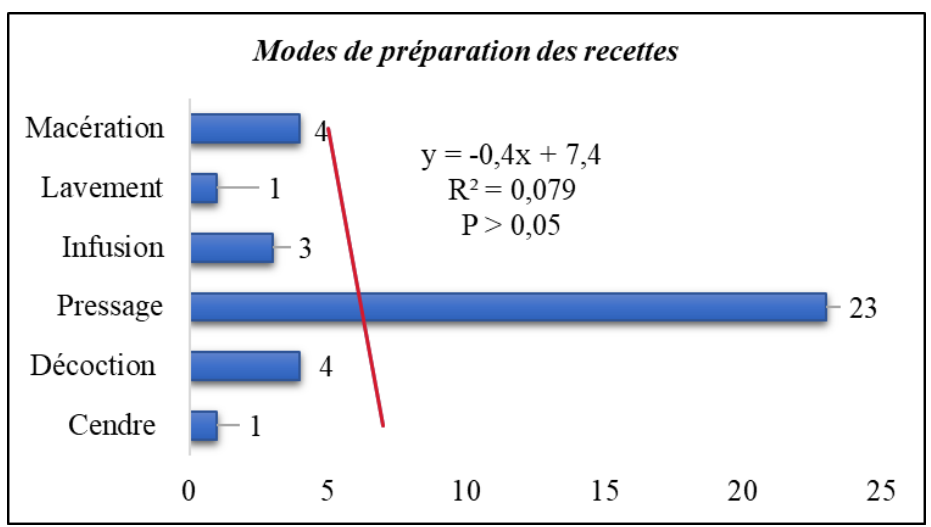

Figure 6 : Variation des modes de préparation des recettes

L'opération consiste à extraire la drogue ou le principe actif (molécule thérapeutique ou curatif) végétale sous une forte pression des mains. Les analyses statistiques nous montrent que ces modes de préparation des recettes ne sont pas équitables, et cela est confirmé par les analyses statiques de régression $\left(R^{2}=0,079\right)$, et le $\mathrm{P}$-value $>0,05$ qui montrent que le pressage est le mode de préparation des recettes le plus utilisé par les Femmes autochtones «Batwa » enceintes que les autres modes de préparation de remèdes. Ainsi, il n'y a pas d'équilibre dans le mode de préparation des recettes chez ce peuple.

\section{Discussion}

\section{- Perception des plantes chez les Batwa}

Le rift Albertin est une zone biogéographique riche en ressources naturelles (White, 1993). Les savoir-faire associés à cette incroyable biodiversité sont considérables. En effet, depuis leurs ancêtres, les peuples Pygmées (Batwa) de l'hinterland du Parc national de Kahuzi-Biega, utilisent les ressources naturelles comme une source de nourriture, de matériaux de construction, d'artisanat, de musique, de jeux et des soins. Selon les femmes autochtones Pygmées (Batwa) de l'hinterland du Parc National de KahuziBiega, les plantes médicinales jouent un rôle particulier et depuis leurs ancêtres, les Pygmées Batwa se transmettent les connaissances sur ces plantes de génération en génération.

Ces plantes médicinales sont des éléments du vivant dont l'homme fait usage pour se soigner depuis ses aïeux (Le Bigot, 2012 ; Delpas \& Johnson, 2014). Elles occupent donc une place particulière dans l'articulation entre la nature et la culture. Leurs usages dépendent à la fois de la perception de la nature et des végétaux et aussi de la perception du corps et de la maladie (Johnson, 2011). 
Chez le peuple Batwa, la maladie et la nature interprètent certains symptômes par la rupture d'un interdit, c'est-à-dire selon les femmes Pygmées, la prescription des plantes pour soigner les symptômes, associée à un système de catégorisation des éléments de la nature qualifié d'«animiste». Cette interprétation donne une place particulièrement importante aux esprits de la nature, plus ou moins puissants, en fonction des éléments concernés. (Cunningham, 1991 ; Fleury et al., 2011).

\section{Importance des plantes thérapeutiques}

Selon le rapport de l'OMS en 2009, environ seulement $45 \%$ des femmes enceintes vont en consultation prénatale. Dans les pays en développement, ce taux oscille entre 60 à $90 \%$ tandis que dans les pays développés, c'est environ 97 à 99\% des femmes enceintes qui vont en consultation prénatale (Breton et al., 2013).

Dans la région du Kivu, les chiffres de 2018 concernant les consultations prénatales avoisinaient les 39\% (DPS, 2019). Ce taux varie d'une région à l'autre selon que les centres de santé sont accessibles ou pas. Nous constatons comme le DPS (2019) qu'en raison de l'éloignement des centres de santé et de la pauvreté en milieu rural, de nombreuses femmes font recours aux pratiques traditionnelles comme leurs congénères Batwa par usage des plantes médicinales durant la grossesse.

L'utilisation de la phytothérapie durant la grossesse est pratiquée par près de $82,30 \%$ de femmes dans le monde (Lisha \& Shantakumar, 2015). En Côte d'Ivoire, malgré le suivi prénatal moderne, la plupart des femmes enceintes utilisent la médecine traditionnelle pour le bon développement du fœtus et pour faciliter l'accouchement (Malan \& Neuba, 2011 ; Kouassi et al., 2017).

Les problèmes obstétricaux sont considérés comme la deuxième catégorie de pathologies la plus traitée dans la région de Kivu montagneux après le paludisme chez les femmes enceintes, d'après des sources des centres de Santé de milieux ruraux dans de l'hinterland du Parc National de KahuziBiega (Balagizi et al., 2007).

Cette pratique, la phytothérapie durant la grossesse est pratiquée par près de $82,30 \%$ de femmes et ce n'est pas seulement chez le peuple Batwa. Dans cet l'hinterland, bien que le suivi de la grossesse à l'aide de la médecine moderne est effectué, la plupart des femmes enceintes utilisent la médecine traditionnelle pour le bon développement du fœtus et pour faciliter l'accouchement (Balagizi et al., 2007).

\section{- Diminution de la transmission intergénérationnelle}

La richesse culturelle subit, tout comme les plantes médicinales, des pressions qui risquent à terme de la faire disparaître. Notre équipe a pu constater une 
certaine pression de la religion et des sectes sur les traditions liées aux plantes, surtout en ce qui concerne la médecine traditionnelle. Il est aussi probable que la migration rurale-urbaine contribue à la perte des savoirs des hommes de l'écosystème d'origine (OMS, 2017).

A cause des répercussions des préjugés des Eglises et de certains personnels de santé, beaucoup de matrones (sages-femmes) et tradipraticiens ne transmettent plus leurs savoirs à leurs descendants et leurs connaissances disparaissent (ex : savoirs liés aux plantes, mais aussi magicoreligieux : divination, esprits guérisseurs, êtres surnaturels guérisseurs ...).

Les détenteurs des savoirs, dont les tradipraticiens, malgré leur enthousiasme à vouloir valoriser la médecine naturelle, se sentent impuissants par rapport aux influences de certaines églises qui considèrent la médecine traditionnelle comme le travail de Satan, donc la pratiquer étant considéré comme un péché. Pour éviter que le patrimoine relatif aux plantes médicinales ne tombe dans l'oubli, notamment par la fragilité de l'oralité de sa transmission, des structures recensent et publient des recueils ethnobotaniques présentant par exemple des monographies de plantes médicinales utilisées par le peuple autochtone.

\section{- Connaissance des plantes médicinales}

L'enquête ethnobotanique réalisée dans l'hinterland de la partie haute altitude du PNKB a inventorié 35 espèces de plantes appartenant à 22 familles intervenant dans le traitement des différentes maladies chez les femmes autochtones riveraines Batwa. L'utilisation des espèces de ces familles de plantes peut être associée à la présence des substances bioactives intervenant dans la santé de la reproduction (Shalukoma et al. 2015, Tsobou et al. 2016, Kabena et al. 2018). Toutefois le mode de préparation et la partie utilisée de la plante diffèrent d'un milieu à l'autre.

L'espèce Aframomum laurenti de la famille de Zingiberaceae, est utilisée pour les maux de constipation par les femmes Batwa dans l'hinterland de la partie de haute altitude du PNKB. Cette utilisation diffère des résultats d'une étude menée par Kabena et al. (2018), qui eux dans leur étude sur les femmes autochtones Pygmées de Mbandaka (à environ 2000 kilomètres à vol d'oiseaux du PNKB), où plutôt cette plante est utilisée pour le traitement des polyménorrhées.

Par ailleurs, cette même espèce est utilisée par les tradipraticiens de la partie montagneuse du PNKB contre les troubles de la grossesse (Shalukoma et al., 2015). Aussi, pour l'Ageratum conyzoides utilisé par les femmes autochtones Pygmées de Mbandaka pour les appétits sexuels (Kabena et al. 2018), ses feuilles et sa tige sont utilisées chez les femmes Batwa riveraines du PNKB pour les maux de ventre et des troubles de la grossesse. 
Certaines espèces sont utilisées pour certaines pathologies par les peuples d'autres régions. Par exemple, la plante Newtonia buchananii utilisée par les tradipraticiens de la région montagneuse de Kahuzi-Biega pour les troubles d'anémie, Planta gopalmata pour les troubles génito-urinaires et le Rumex bequaertii pour les infections (Shalukoma et al., 2015). Mais les mêmes plantes sont utilisées aussi chez les femmes enceintes autochtones Batwa riveraines du PNKB de la région montagneuse similaire pour soigner les maladies dues aux plaintes liées aux vertiges, aux anémies et autres menaces d'avortement.

Ainsi, les plantes médicinales sont perçues de manière très contrastée selon les différents protagonistes et usagers de la nature (Mangambu, 2016) : pour les autochtones de riveraine du PNKB, elles font sens dans une approche spirituelle de la nature, tandis que pour de nombreux autres acteurs, notamment dans le domaine pharmaceutique, elles sont perçues comme des usines à molécules bioactives, ou encore à travers leur potentiel économique (Mangambu et al., 2012 et 2015b).

\section{Conclusion}

Les avantages de la médecine traditionnelle, en dépit de son manque de caractère empirique, restent bien considérables. Nous venons de constater que les femmes autochtones riveraines Batwa ayant acquis cette pratique ancestrale n'ont besoin d'aucune vulgarisation ou propagande pour son acceptation. C'est une médecine qui demeure à la portée de tous dans la perspective du temps et de l'espace par rapport aux produits utilisés dans la médecine moderne qui s'avèrent généralement plus chers.

Dans cette étude, 35 espèces de plantes médicinales sont signalées pour la première fois par les femmes autochtones Batwa enceintes riveraines de la partie haute altitude du Parc National de Kahuzi Biega (PNKB). Elles sont citées comme étant susceptibles de soigner une vingtaine de maladies humaines chez les femmes autochtones Batwa enceintes. L'étude révèle les organes des plantes médicinales utilisés, leur fréquence d'utilisation, leur mode de préparation, le type de leur morphologie.

Les savoirs de la population autochtone sur l'utilisation des plantes médicinales sont des savoirs non écrits. Pour éviter toute perte de ces connaissances, il est nécessaire de les cataloguer et de les archiver. Le recensement des savoirs traditionnels semble répondre à un objectif louable : éviter qu'ils ne se perdent

Pour cette raison, nous pensons que l'état Congolais et la Province du Sud-Kivu, les Gestionnaires du Parc peuvent s'impliquer pour valoriser ses connaissances médicinales, mettre au point des stratégies pour préserver et conserver ces plantes ex situ pour un accès facile. 


\section{References:}

1. Ahmed, S. M., Nordeng, H., Sundby, J., Aragaw, Y. A., \& de Boer, H. J. (2018). The use of medicinal plants by pregnant women in Africa: a systematic review. Journal of ethnopharmacology, 224, 297-313.

2. Amisi F., Ilambu O., Liengola I., Kujirakwinja D., Hart J., Grossman F. \& Plumptre A. (2008). The impact of the wars on Kahuzi-Biega National Park. Results of surveys between 2000 - 2008. Unpublished report, Wildlife Conservation Society, 1-31.

3. APG IV. An update of Angiosperm Phylogeny Group classification for the orders and families of flowering. Botanical Journal of the Linnean Society. 2016; 181: 1-20. https://doi.org/10.1111/boj.12385

4. Bafor, E. E. (2017). Potentiels d'utilisation des plantes médicinales dans les troubles de la reproduction chez la femme-La voie à suivre. African Journal of Reproductive Health, 21(4), 12.

5. Balagizi I., Ratti E., Kambale V. (2007). Les plantes médicinales du Bushi. Gênes, Italie, A. Insieme al Terzo Mondo, 313 p

6. Bernstein, J., Roy, E. and Bantong, B. (1997). The use of plot surveys for the study of ethnobotanical knowledge: a bruneidusun example. Journal of Ethnobiological., 17: 1, 69-96

7. Bloesch, U, Troupin, G. et Derungs, N. (2009). Les plantes ligneuse du Rwanda : Flore, écologie et Usage. Ed. Shaker Verlag, Berlin/Allemagne, $760 \mathrm{p}$.

8. Chibembe, A.S.; Birhashirwa, N R.; Kamwanga, F.F. \& Mangambu M. (2015). Exploitation de Bambous (Sinarundinaria alpina ( $K$. Schum.) C.S. Chao et Renvoize), cause des conflits entre le Parc National de Kahuzi-Biega et la population environnante: stratégie de conservation et de résolution de Conflit. International Journal of Environmental $\quad$ Studies, $265-284$. http://dx.doi.org/10.1080/00207233.2014.961270

9. Cunningham, A. B. (1991). Development of a conservation policy on commercially exploited medicinal plants: a case study from southern Africa. Conservation of medicinal plants, 337.

10. De Caluwé, E., De Smedt, S., Assogbadjo, A.E., Samson, R., Sinsin, B. and Van Damme, P. (2009). Ethnic differences in use value and use patterns of baobab (Adansoniadigitata L.) in northern Benin. Africa Journal of Economy, 47: 433-440.

11. Defour G. (1995). Éléments d'identification de 400 plantes médicinales vétérinaires du Bushi. Bukavu, Zaïre, Ban- dari, 125 p.

12. Delpas C. et Johnson P.W. (2014). Protéger les savoirs des peuples autochtones, Le monde diplomatique, Janvier, 12-13. 
13. Fleury M., Karpe P., Et Tiouka A. (2011).Valorisation des produits forestiers et construction d'un patrimoine guyanais : articulation entre le social, le biologique et le juridique ", MAM LAM FOUCK, Serge \& Isabelle HIDAIR (sous la dir. de), La question du patrimoine en Guyane, Matoury, Ibis rouge éditions, 191-215.

14. Gururani, S. (2002). Le savoir des femmes du tiers monde dans le discours sur le développement. Revue internationale des Sciences Sociales, (3), 353-363.

15. Heinrich, M., Ankli, A., Frei, B., Weimann, C., \&Sticher, O. (1998). Medicinal plants in Mexico: Healers' consensus andculturalimportance. SocialScience \& Medicine, Friedman, J., Yaniv, Z., Dafni, A., \&Palewitch, D. (1986). A preliminary classification of the healing potential of medicinal plants, based on a rational analysis of an ethnopharmacological field survey among Bedouins in the Negev Desert, Israel. Journal of Ethnopharmacology, 16(2-3), 275-287. https://doi.org/10.1016/0378-8741(86)90094-2

16. Imani, M. ; Zapfack, L. ; Mangambu, M. ; Penedimanja, P. ; Mwanga Mwanga, I. ; Boyemba F., \& Amani, C. (2016).Caractérisations Structurale, Floristique et biomasse ligneuse du peuplement À Hagenia abyssinicadu Parc National de Kahuzi-Biega (Rift Albertin, RDC). $\begin{array}{llll}\text { European Scientific Journal, } 12 & \text { (15) : 198-209 }\end{array}$ http://dx.doi.org/10.19044/esj.2016.v12n15p189

17. Johnson P.W. (2011). Biopiraterie. Quelles alternatives au pillage des ressources naturelles et des savoirs ancestraux ? Paris, Charles Léopold Mayer, $239 \mathrm{p}$.

18. Kabena N.O., Katunda M.R., Bikandu K., Botefa I., Ngombe K., Mpiana. T., Mboloko E., Lukoki L. (2018). Étude ethnobotanique des plantes utilisées par les Pygmées pour la santé de la reproduction à Mbandaka et ses environs / Province de l'Equateur, RD Congo. International Journal of Innovation and Scientific Research, 36 (1), 19-29

19. Le Bigot J. (2012). Focus on Medicinal Plants in Madagascar. Medicinal Plant Conservation, 15, 25-29.

20. Lebrun J \&Stork A (1991), Enumération des plantes à fleurs d'Afrique tropicale. Conservatoire et Jardin botaniques de la Ville de Genève, Suisse, 1-243

21. Lebrun J.-P. \&Stork A-L (1997), Enumération des plantes à fleurs d'Afrique Tropicale. Conservatoire et Jardin botaniques de la Ville de Genève, Genève, Suisse, 1-712 Kakudidi, E. (2004). Cultural and social uses of plants from and around Kibale National Park, Western Uganda. Afr. J. Ec., 42 (1): 114-118. Kamini S., (2007). Ethnobotanical Studies of Some Important, 11: 164 -172. 
22. Lebrun J-P \&Stork A-L (1995), Enumération des plantes à fleurs d'Afrique tropicale. Conservatoire et Jardin botaniques de la Ville de Genève, Suisse, 1-341

23. Malay, B. (2011). Ethno medicinal importance of some common Pteridophytes used by tribals of Ranchi and Latehar district of Jharkhand, India. The international quarterly journal of ethno and social sciences, 3 (1): 5-8.

24. Mangambu M. J., Diggelen R., MwangaMwanga J. C., Ntahobavuka H., Malaisse F., Robbrecht E. (2012). Étude eth- noptéridologique, évaluation des risques d'extinction et stratégies de conservation aux alentours du Parc National de Kahuzi Biega (RD Congo). Revue GEOECO TROP, 36 : 137-158.

25. Mangambu M (2013).Taxonomie, Biogéographie et Ecologie des Ptéridophytes de l'écosystème forestier des montagnes du Parc National de Kahuzi-Biega à l'Est de la R.D. Congo. Thèse de doctorat, Université d'Anvers/Belgique, $463 \mathrm{p}$.

26. https://www.uantwerpen.be/en/rg/ecobe/research/phd/

27. Mangambu, M.; Aluma, K.; van Diggelen, R.; Rugenda-Banga, R.; Mushangalusa, K., Chibembe, S.; Ntahobavuka, H.; Nishuli, B. \&Robbrecht, E. (2015a). Etudes ethnobotanique et ethnolinguistique des ressources forestières ligneuses utilisées par la population du couloir écologique du parc national de Kahuzi-Biega (R D. Congo). European Scientific Journal, 11 (15) : 135-162, http://dx.doi.org/10.19044/esj.2014.v.183

28. Mangambu, M.; Muyisa, K.; Nishuli, B.R. \&Ntahobavuka, H. (2015b). Utilisation des ressources forestières ligneuses par la population habitant la zone submontagnarde du Parc National de Kahuzi-Biega (R.D. Congo). International Journal of Innovation and Applied Studies, $11 \quad$ (2): 508-521. http://ijias.issrjournal.org/abs.php?article=IJIAS-15-094-09

29. Mangambu, M. (2016). Diversité, Biogéographie et Ecologie des Ptéridophytes : Cas de massif montagneux du Parc National de Kahuzi-Biega à l'Est de la RD. Congo. Editions universitaires européennes, $354 \mathrm{p}$.

30. Mangambu, M.; Ntahobavuka, H.; Basinyize, B.; Lokumu, I. \&Robbrecht, E. (2018). Pteridaie: indicator of the vegetation succession and dynamics in the forests Chablis and Edges in KahuziBiega National Park (Eastern D. R. Congo, Albertine Rift). International Journal of Biological Research, 6 (1) 5-13, http://dx.doi.org/10.14419/ijbr.v6i1.9241

31. MangambuM. et Kambale K. (2019).Pratiquestraditionnelles et culturelles des communautéslocales en matière de la conservation de 
la biodiversité

cas dans la Réserve Naturelle d'Itombwe à 1'Est de la RD Congo. Network, Workhop Bukavu, 1-23 p

32. Maregesi, S., Ngassapa, O., Pieters, L et Vlietinck, A. (2007). Ethnopharmacological survey of the Bunda district, Tanzania: Plants used to treat infectious diseases. Journal of Ethnopharmacology, 113: 457-470.

33. Mubalama K., Igunzi A., Banswe T., Asesa L. et KambaleK. (2018). Savoirs traditionnels conciliés aux connaissances scientifiques comme nouveau paradigme de la conservation des Aires Protégées : Cas de Malambo (Réserve Naturelle d'Itombwe, RD Congo). Annales des Sciences et des Sciences Appliquées, vol. 4(3), 129-167

34. OMS 'Organisation Mondiale de la Santé' (2017). Recommandation de l'OMS concernant les soins prénatals pour que la grossesse soit une expérience positive. $158 \mathrm{p}$

35. Pourchez, L. (2011). Savoirs des femmes : médecine traditionnelle et nature - Maurice, Réunion, Rodrigues. Savoirs locaux et autochtones, 1. UNESCO : Paris, $120 \mathrm{p}$.

36. Schneider E. (1996). Contribution à l'étude de l'ethnobotanique et de la médecine traditionnelle du Bushi (Kivu, Zaïre). Anthropos, 91: 5374.

37. Shalukoma, C. (2008). Usage des plantes médicinales par les populations riveraines de la partie de haute altitude du Parc National de Kahuzi-Biega, Sud /Kivu, République Démocratique du Congo. Mémoire de DEA Université libre de Bruxelles 67p + Annexes

38. Shalukoma, C., Bogaert, J., Duez, P., Stévigny, C., Pongombo, C., \&Visser, M. (2015). Les plantes médicinales de la région montagneuse de Kahuzi-Biega en République démocratique du Congo: Utilisation, accessibilité et consensus des tradipraticiens. Bois et forêts des tropiques, (326), 43-55.

39. Tabuti J.R.S., Lye K.A. \&Dhillion S.S. (2003).Traditional herbal drugs of Bulamogi, Uganda: plants, use and administration. Journal of Ethnopharmacology, 88, 19-44.

40. Tardío, J., \&Pardo-de-Santayana, M. (2008). CulturalImportance Indices : A Comparative Analysis Based on the Useful Wild Plants of Southern Cantabria (Northern Spain). Economic Botany, 62(1), 24-39. https://doi.org/10.1007/s12231-007-9004-5

41. Tsobou, R., Mapongmetsem, P. M., \& Van Damme, P. (2016). Medicinal plants used for treating reproductive health care problems in Cameroon, Central Africa. Economic botany, 70 (2), 145 - 159.

42. Yao, K., Koné, M. W., \&Kamanzi, K. (2015). Contribution des LégumesFeuilles à la Nutrition des Populations en Zones Urbaines de 
la Côted'Ivoire. European Journal of Scientific Research, 130(4), 338 -351 .

43. White F., (1993). The AETFAT chorological classification of Africa: history, methods and applications. Bulletin van de National Plantentuin van België, 62: 225 - 281. 